\title{
A megatoothed shark (Carcharocles angustidens) nursery in the Oligocene Charleston Embayment, South Carolina, USA
}

\author{
Addison E. Miller, Matthew L. Gibson, and Robert W. Boessenecker
}

\begin{abstract}
Many extant sharks are cosmopolitan as adults but inhabit nursery areas as youngsters - often shallow, dynamic ecosystems with abundant prey for neonates and juveniles. Megatoothed sharks (Otodontidae) were the largest sharks of all time, and nursery areas have been demonstrated for Carcharocles megalodon in the Miocene of Panama, Spain, Florida, and Maryland. An earlier study hypothesized a nursery area for Carcharocles angustidens in the upper Oligocene (23-25 Ma) Chandler Bridge Formation of Charleston, South Carolina. We tested this by reporting and analyzing two collections $(n=127)$ dominated by small teeth of $C$. angustidens from the Chandler Bridge Formation and some teeth from the underlying lower Oligocene (29-26.57 Ma) Ashley Formation ( $n=9$ ). Correcting for tooth position, published body length estimation equations yielded body length estimates of $1.5-6.5 \mathrm{~m}$ for most individuals. Size-based assignment to age classes (neonates, juveniles, adults) is modified from the larger $C$. megalodon and scaled based on the largest available specimens of $C$. angustidens, reported herein. These assemblages are dominated by small individuals (juveniles and neonates) and include few adults. The Oligocene Charleston embayment therefore represents the first documented paleo-nursery area for $C$. angustidens.
\end{abstract}

Addison Miller. Department of Geology and Environmental Geosciences, College of Charleston, Charleston, South Carolina 29424, USA. addisonemiller12@gmail.com

Matthew Gibson. Charleston Museum, Charleston, South Carolina 29403, USA.

mgibson@charlestonmuseum.org

Robert Boessenecker, Mace Brown Museum of Natural History and Department of Geology and Environmental Geosciences, College of Charleston, Charleston, South Carolina 29424, USA.

Corresponding Author. boesseneckerrw@cofc.edu

Keywords: sharks; Otodontidae; Paleogene; Cenozoic; paleoecology; Chondrichthyes

Submission: 31 December 2020. Acceptance: 19 May 2021.

Miller, Addison E., Gibson, Matthew L., and Boessenecker, Robert W. 2021. A megatoothed shark (Carcharocles angustidens) nursery in the Oligocene Charleston Embayment, South Carolina, USA. Palaeontologia Electronica, 24(2):a19. https://doi.org/ $10.26879 / 1148$

palaeo-electronica.org/content/2021/3372-oligocene-shark-nursery

Copyright: June 2021 Paleontological Society.

This is an open access article distributed under the terms of Attribution-NonCommercial-ShareAlike 4.0 International (CC BY-NC-SA 4.0 ), which permits users to copy and redistribute the material in any medium or format, provided it is not used for commercial purposes and the original author and source are credited, with indications if any changes are made. creativecommons.org/licenses/by-nc-sa/4.0/ 


\section{INTRODUCTION}

Otodontid sharks are giant extinct predatory sharks found worldwide from the Cretaceous through the Pliocene, including the prominent Carcharocles lineage terminating with the extinction of Carcharocles megalodon 3.5 Ma (Gottfried et al., 1996; Purdy, 1996; Purdy et al., 2001; Kent, 2018). Carcharocles evolved from Otodus obliquus during the Eocene through the development of serrations, an adaptation for feeding upon newly evolved marine mammals with thick blubber (Frazzetta, 1988; Abler, 1992), which first became globally widespread during the middle Eocene. A megatoothed shark chronospecies from the Oligocene, Carcharocles angustidens, is found worldwide (Atlantic Coastal Plain, USA; Belgium; France; Japan; Ecuador; Australia; New Zealand). Adult size of 8-9 m body length was estimated for C. angustidens, based on a partial skeleton from New Zealand (Gottfried and Fordyce, 2001). Teeth of $C$. angustidens are triangular, serrated, bear serrated lateral cusplets, and a robust U-shaped root with triangular lobes (Gottfried and Fordyce, 2001). Bite marks on fossil marine mammal bones are frequently attributed to Carcharocles (Purdy, 1996; Aguilera et al., 2008; Kallal et al., 2010; Collareta et al., 2017; Godfrey et al., 2018).

Many species of modern sharks return to particular locations to pup that are favorable for the growth of juveniles (Castro, 1993; Heithaus, 2007). Shark nurseries are often found in protected shallow marine embayments lacking larger sharks, therefore offering protection, whereas others are on the open coast (Heithaus, 2007; Heupel et al., 2007). In addition to offering protection from potential predators, nurseries are typically rich in small prey items (Castro, 1993; Heithaus, 2007). Multiple species may use the same nursery area (Castro, 1993). Many ancient examples of shark nurseries have been interpreted from the Cenozoic fossil record, based on a preponderance of juvenile or neonate teeth, evidence of protected shallow marine settings, and abundant potential prey or high primary productivity (Pimiento et al., 2010; Landini et al., 2017; Herraiz et al., 2020; Villafaña et al., 2020). Nurseries for the megatoothed shark C. megalodon have been reported from Miocene and Pliocene assemblages in Panama, Spain, and the Atlantic coast of the USA (Maryland and Florida; Pimiento et al., 2010; Herraiz et al., 2020).

Carcharocles angustidens teeth are frequently collected in Oligocene deposits of the Charleston embayment in South Carolina (Figure 1), which has yielded a diverse assemblage of marine verte- brates including sharks, bony fish, sea turtles, an estuarine crocodile, sea birds, sea cows, and early whales and dolphins (Boessenecker and Geisler, 2018, and references therein). Sea turtles, early dolphins (Odontoceti), and baleen whales made for ample prey (Purdy, 1996; Sanders et al., 1982). Excavation of a bonebed in the Chandler Bridge Formation with a large sample ("about 100") of $C$. angustidens dominated by small teeth interpreted as juveniles and rare large teeth representing adults led Purdy (1996) to propose that the Charleston embayment hosted a megatoothed shark nursery during the Oligocene. We evaluate the shark nursery hypothesis through formal study of this bonebed sample and also report a newly discovered second sample of megatoothed sharks from the same strata.

\section{Institutional Abbreviations}

CCNHM, Mace Brown Museum of Natural History, College of Charleston, Charleston, South Carolina, USA; ChM, Charleston Museum, Charleston, South Carolina, USA; OU, University of Otago Geology Museum, Dunedin, New Zealand.

\section{GEOLOGIC BACKGROUND}

The specimens in this study were collected from two localities in the vicinity of Ladson and Summerville, South Carolina, from the Oligocene Chandler Bridge and Ashley formations (Figure 1). The Ashley Formation is $10-25 \mathrm{~m}$ thick, made up of olive-tan sandy limestone, and has been dated to 28-29 Ma using ${ }^{87} \mathrm{Sr} / 86 \mathrm{Sr}$ ratios (Rupelian equivalent; Weems et al., 2016; Boessenecker and Fordyce, 2017). The Ashley Formation is mainly calcareous, besides the basal phosphatic bed. The Ashley Formation is made up of three members: the Gettysville Member, the Runnymede Marl Member, and the Givhan's Ferry Member. The Gettysville Member has only been recorded in a single core, but the Runnymede Marl and Givhan's Ferry Members are commonly exposed in excavations around the Charleston area. The Runnymede Marl Member is a pale green-gray calcarenite with abundant burrows, shells, vertebrate skeletal fragments, and phosphate nodules. The Givhan's Ferry Member consists of olive brown glauconitic calcarenite (R.W. Boessenecker, personal obs.) that is more fossiliferous than the Runnymede Marl Member. The basal contact of the Givhan's Ferry Member is a diffuse, bioturbated boundary with pods of mollusk and barnacle shell fragments, phosphate pebbles, and vertebrate elements forming burrow infill. 

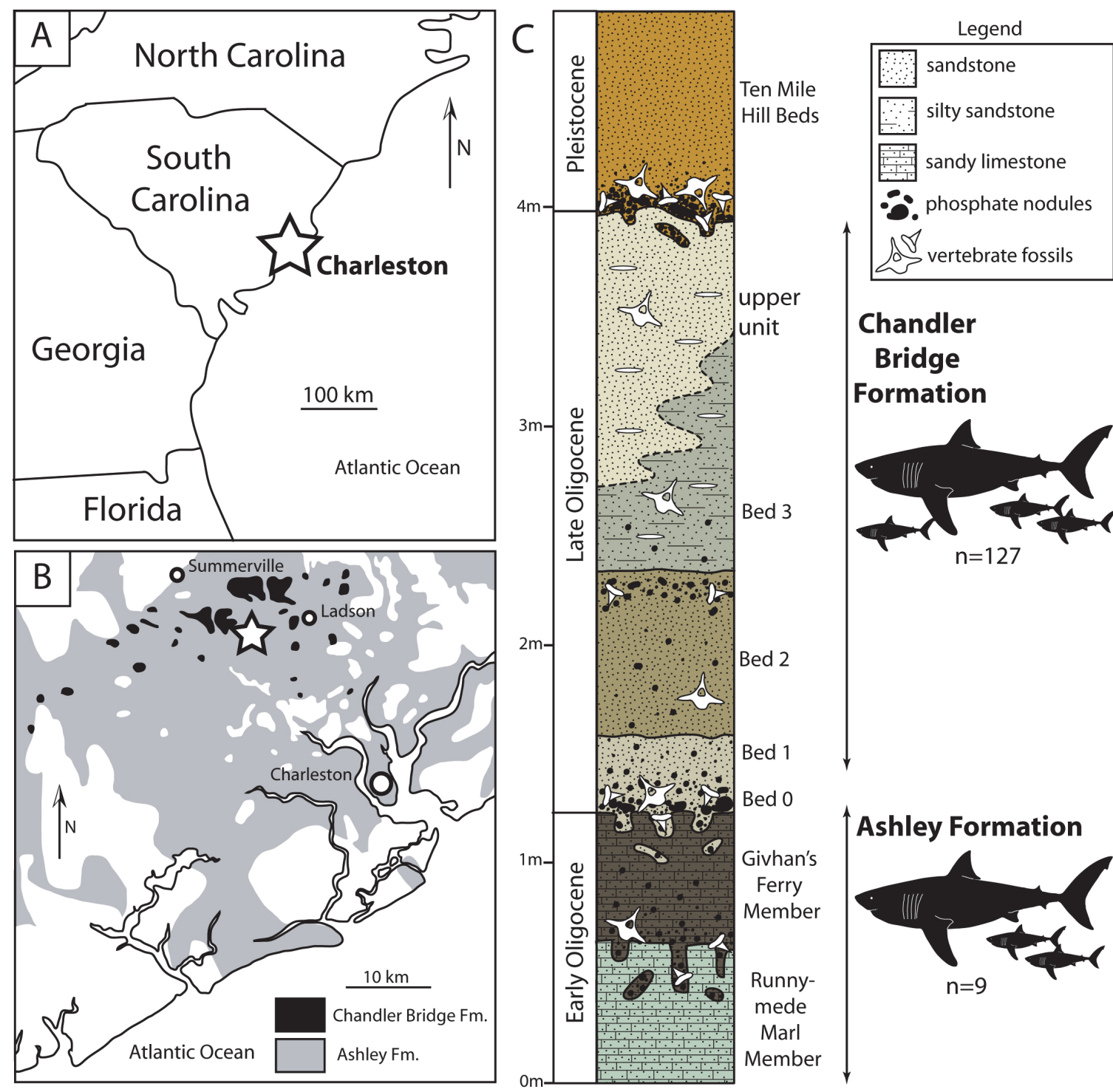

\section{Chandler \\ Bridge}

Formation
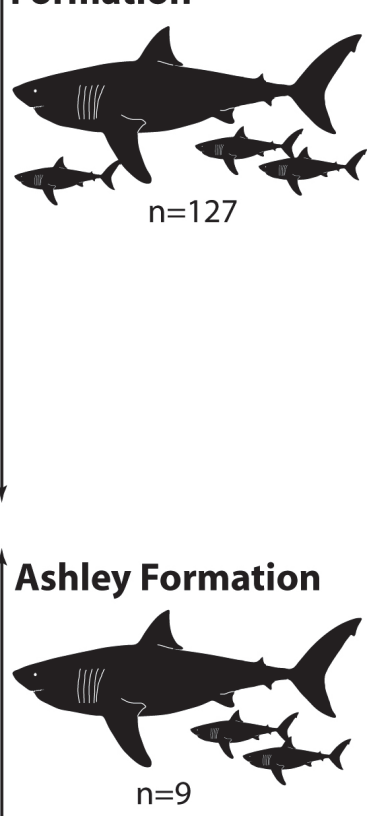

FIGURE 1. Generalized geology and stratigraphy of Oligocene marine strata in the vicinity of Ladson and Summerville, South Carolina, USA. A) Map of South Carolina showing Charleston. B) Map of Charleston showing the extent of Oligocene marine rocks; star denotes the location of the McKewn subdivision and Chandler Bridge excavation localities. C) Stratigraphic column of Oligocene marine rocks and overlying deposits as exposed in stormwater pond excavations at the McKewn Subdivision locality near Ladson, South Carolina. Modified from Fallon and Boessenecker (2020).

Most specimens in this study were collected from the Chandler Bridge Formation, which overlies the Ashley Formation. The Chandler Bridge Formation is typically thin $(30-100 \mathrm{~cm}$ at most localities) but is $2.5 \mathrm{~m}$ thick at the McKewn subdivision locality. The Chandler Bridge is upper Chattian and is dated to 24.7-23.5 Ma based upon dinoflagellates and strontium isotopes (Weems et al., 2016; Boessenecker and Fordyce, 2017, and references therein). This large range in thickness and patchiness is most likely caused by later Neogene erosion (Katuna et al., 1997). The Chandler

Bridge Formation consists of several beds composed chiefly of tan, brown, and olive, richly fossiliferous, unlithified, massively bedded, fine-very fine sandstone and siltstone. A phosphatic bonebed typically mantles the lower contact, though vertebrates are common throughout the unit. At the McKewn subdivision locality, the basal bonebed is rich in large, brown, irregularly-shaped nodules, and there is a second bonebed $70 \mathrm{~cm}$ higher that is less richly fossiliferous but dominated by tan to light gray phosphate nodules $1-3 \mathrm{~cm}$ in diameter. A more detailed description of the stratigraphy at this 
locality is provided by McCuen et al. (2020). All specimens collected from the McKewn subdivision locality originated from beds 1 and 2 (and possibly bed 0 ) of the Chandler Bridge Formation, whereas at the Chandler Bridge excavation, most were derived from bed 3 (Sanders, 1980).

A well-established vertebrate assemblage from the Chandler Bridge Formation includes bony fish, marine birds, marine mammals, crocodiles, and most important for this study, sharks (Sanders et al. 1982, Boessenecker and Geisler 2018, and references therein). Fossil bony fish and sharks suggest that the Chandler Bridge Formation was deposited under constant open marine conditions (Cicimurri and Knight, 2009), in contrast to earlier estuarine interpretations (Katuna et al., 1997). The shark assemblage in particular suggests inner to middle shelf environments (Cicimurri and Knight, 2009).

\section{MATERIALS AND METHODS}

Teeth were identified to tooth position based on the reconstructed dentition of Carcharocles angustidens reported by Gottfried and Fordyce (2001). Crown height and width (after Pimiento et al., 2010: figure S2) was measured to the nearest tenth of a millimeter using digital calipers. Based on tooth position, body length was estimated using regression equations derived from tooth size to body length data for extant Carcharodon carcharias analyzed by Shimada (2002). In the absence of complete skeletons permitting measurement of body length, Carcharodon carcharias is a reasonable modern analog for reconstructing body length from otodontid tooth dimensions (Pimiento et al., 2010). Note, however, that a recent study suggests that these estimates might underestimate the maximum body length, and that posterior teeth overestimate body length versus anterior teeth (Perez et al., 2021). Because the goal of this study is to evaluate the relative size, and not evaluate different methods, we follow Pimiento et al. (2010) and Shimada et al. (2020) in using the equations from Shimada (2002). Photographs were taken with a Canon Rebel T5 and a Tokina f/2.8 100 mm macro lens.

Pimiento et al. (2010) and Gottfried et al. (1996) demarcated growth stage bins for three age classes in Carcharocles megalodon: neonates, juveniles, and adults. Pimiento et al. (2010) and Gottfried et al. (1996) identified neonates as individuals less than $4 \mathrm{~m}$ reconstructed body length, juveniles between $4 \mathrm{~m}$ and $10.5 \mathrm{~m}$, and adults over $10.5 \mathrm{~m}$. However, Carcharocles angustidens is smaller than C. megalodon, and these size bins need adjustment. Gottfried and Fordyce (2001) identified the maximum length of OU $22261 \mathrm{C}$. angustidens to be $9.3 \mathrm{~m}$. However, we recalculated this using Shimada's (2002) regression-derived equations using the largest tooth of OU 22261 (Gottfried and Fordyce, 2001:fig. 3a), and found a slightly smaller body length of $8.46-8.81 \mathrm{~m}$ (depending upon the A1 or A2 tooth position, A1 being upper first anterior).

Because Purdy (1996) referred to teeth of even larger Carcharocles angustidens specimens rivaling teeth of Carcharocles megalodon in size, we searched collections at CCNHM and ChM for large specimens from other localities in South Carolina to reconstruct the maximum size of $C$. angustidens. We located several specimens exceeding $75 \mathrm{~mm}$ crown height ( $\mathrm{n}=6$; Figure 2, Table 1). The largest single specimen we could locate is ChM PV 7281 , an isolated A1 lacking locality data but from the Charleston area. Using Shimada's (2002) equation for the $A 1$ tooth position, we calculated the body length of ChM PV 7281 (Figure 2; Table 1) as $11.22 \mathrm{~m}$. Using a maximum length of $17 \mathrm{~m}$ for C. megalodon (after Pimiento et al., 2010), and $11.2 \mathrm{~m}$ for $C$. angustidens based on the specimen ChM PV 7267, we estimated that $C$. angustidens had a maximum length $65 \%$ of the length of $C$. megalodon and applied a $65 \%$ correction to these growth stage bins (Pimiento et al., 2010; Gottfried et al., 1996) and consider $C$. angustidens neonates to be less than $2.6 \mathrm{~m}$, juveniles between $2.6 \mathrm{~m}$ and $6.8 \mathrm{~m}$, and adults more than $6.8 \mathrm{~m}$. Isometric scaling of these growth stage bin boundaries is defensible on the grounds that lamniform and carcharhiniform sharks do not exhibit allometric growth (Irschick and Hammerschlag, 2014; Irschick et al., 2017; Cooper et al., 2020).

'Ex situ' teeth (discovered after they had been eroded from Oligocene strata, typically found on beaches, dredge spoils, streambeds, and river bottoms) from other localities were only used to estimate the maximum size of $C$. angustidens and were not included in the body size distribution analysis.

Specimens studied here $(n=136)$ originate from three collections made at two localities: the Chandler Bridge Formation at the Chandler Bridge excavation site $(n=95)$, and the Ashley $(n=9$; not included in analyses of the Chandler Bridge samples) and Chandler Bridge formations $(n=32)$ at the McKewn subdivision in the vicinity of Ladson and Summerville, South Carolina. Rather than reporting all specimens of Carcharocles angustidens in 

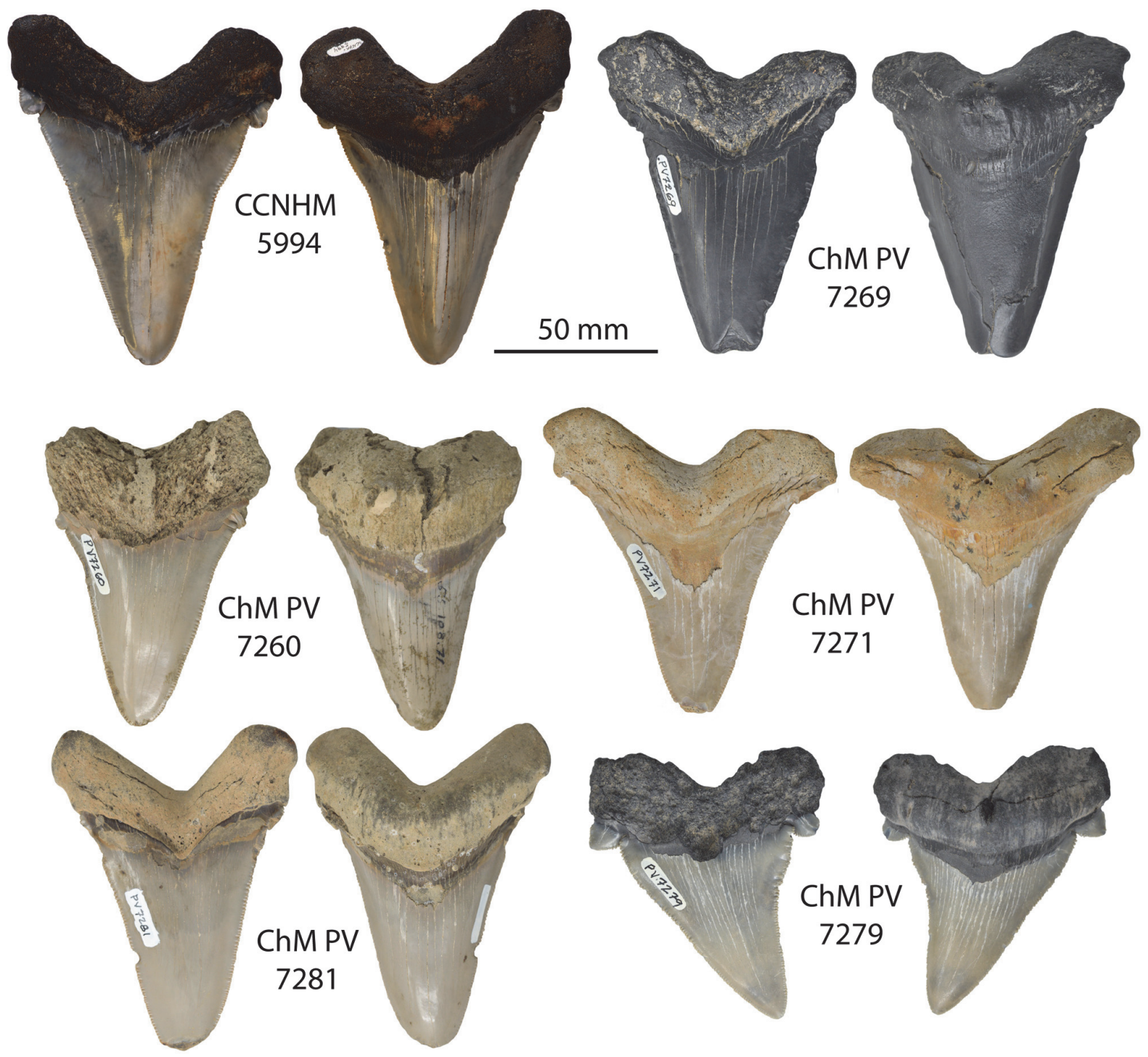

FIGURE 2. The largest available specimens (crown height $>75 \mathrm{~mm}$ ) of Carcharocles angustidens, in labial view (left) and lingual view (right).

museum collections from the area, we restricted the studied sample to these collections to limit collection bias. Collection bias affects fossils at Oligocene exposures near Charleston in a number of ways. Shark tooth diving in the Wando, Cooper, and Ashley rivers is popular, but is obviously biased towards collection of large trophy specimens: 1) murky water with poor visibility limits the ability of divers to find small teeth and biases collection towards larger specimens; 2) larger teeth are more likely to be exposed and recognized on the river bottom, and 3) there is intense desire and competition for collecting large trophy specimens. Further compounding this is the lack of geological context for most river-collected specimens. Collect- ing ex situ specimens from local beaches and spoils along waterways is a popular activity for local collectors and paleontological tourists, who may retrieve the biggest, most complete teeth while leaving the small, incomplete teeth behind. These ex situ collections also lack stratigraphic context. Lastly, private collectors are unlikely to donate an entire collection - even if made in an unbiased fashion from in situ exposures - owing to the commercial and prestige value placed on these coveted teeth by the collecting community.

We instead focused on unbiased samples collected 'in situ' (specimens discovered while still embedded in the rock or by screenwashing, so stratigraphic origin is certain) by field paleontolo- 
TABLE 1. Measurements, identifications, and estimated total lengths of specimens used in this study. " " denotes estimated measurement. Tooth position after Shimada et al. (2003); A=anterior; L=lateral; upper case denotes upper tooth, lower case denotes lower tooth. AF = Ashley Formation; $\mathrm{CB}=$ Chandler Bridge Formation.

\begin{tabular}{|c|c|c|c|c|c|c|}
\hline Specimen & $\mathrm{CW}(\mathrm{mm})$ & $\mathrm{CH}(\mathrm{mm})$ & Tooth Position & $\mathrm{TL}(\mathrm{m})$ & Age class & Formation \\
\hline CCNHM 5173 & 43 & $\sim 40$ & L2-L3 & 5.62 & juvenile & AF, McKewn \\
\hline CCNHM 5175 & 31 & $?$ & $?$ & $?$ & juvenile? & AF, McKewn \\
\hline CCNHM 5187 & 35 & 43 & $\mathrm{~A} 1-\mathrm{A} 3, \mathrm{a} 1-\mathrm{a} 3$ & 6.10 & juvenile & AF, McKewn \\
\hline CCNHM 6081 & 70 & $70-80$ & $?$ & $?$ & adult? & AF, McKewn \\
\hline CCNHM 6082 & 27 & $\sim 22$ & 11 & 3.93 & juvenile & AF, McKewn \\
\hline CCNHM 6083 & 25.4 & $?$ & $?$ & $?$ & juvenile? & AF, McKewn \\
\hline CCNHM 6084 & $\sim 36$ & $?$ & $?$ & $?$ & juvenile? & AF, McKewn \\
\hline CCNHM 6085 & 28 & $\sim 34$ & $\mathrm{~A} 1-\mathrm{A} 3, \mathrm{a} 1-\mathrm{a} 3$ & 4.83 & juvenile & AF, McKewn \\
\hline CCNHM 6087 & 18 & $\sim 23$ & L1-L3, I1-I3 & 4.07 & juvenile & AF, McKewn \\
\hline CCNHM 6086 & 33 & 29 & L1 & 4.24 & juvenile & CB, McKewn \\
\hline CCNHM 5151 & $\sim 42$ & 45 & a2 & 6.04 & juvenile & CB, McKewn \\
\hline CCNHM 5152 & 35 & 41 & a1 & 6.62 & juvenile & CB, McKewn \\
\hline CCNHM 5153 & $\sim 41$ & $\sim 40$ & A2-A3,a2-a3 & 5.9 & juvenile & CB, McKewn \\
\hline CCNHM 5154 & 35 & $\sim 29$ & A3 & 4.76 & juvenile & CB, McKewn \\
\hline CCNHM 5155 & $\sim 44$ & $\sim 34$ & L1 & 4.88 & juvenile & CB, McKewn \\
\hline CCNHM 5156 & $\sim 26$ & 38 & A2-A3,a2-a3 & 5.61 & juvenile & CB, McKewn \\
\hline CCNHM 5157 & 39 & 26 & L4-L5 & 5.72 & juvenile & CB, McKewn \\
\hline CCNHM 5158 & 40 & 38 & L2 & 5.15 & juvenile & CB, McKewn \\
\hline CCNHM 5159 & $\sim 32$ & $\sim 20$ & a2-12 & 3.37 & juvenile & CB, McKewn \\
\hline CCNHM 1560 & 23 & 15 & L5 & 3.9 & juvenile & CB, McKewn \\
\hline CCNHM 1563 & 29 & $\sim 29$ & $\mathrm{~A} 1-\mathrm{A} 3, \mathrm{a} 1-\mathrm{a} 3$ & 4.12 & juvenile & CB, McKewn \\
\hline CCNHM 1564 & $\sim 40$ & $\sim 38$ & L2-L4 & 5.82 & juvenile & CB, McKewn \\
\hline CCNHM 1566 & $\sim 42$ & 39 & L2 & 5.29 & juvenile & CB, McKewn \\
\hline CCNHM 1567 & 40 & 47 & A1 & 5.47 & juvenile & CB, McKewn \\
\hline CCNHM 5168 & $\sim 30$ & $\sim 33$ & $\mathrm{~A} 1-\mathrm{A} 3, \mathrm{a} 1-\mathrm{a} 3$ & 4.69 & juvenile & CB, McKewn \\
\hline CCNHM 5169 & $\sim 15$ & $\sim 25$ & a1-I1 & 3.93 & juvenile & CB, McKewn \\
\hline CCNHM 5170 & $\sim 40$ & $\sim 39$ & $\mathrm{~A} 1-\mathrm{A} 3, \mathrm{a} 1-\mathrm{a} 3$ & 5.55 & juvenile & CB, McKewn \\
\hline CCNHM 5171 & $\sim 34$ & $\sim 31$ & a1-I1 & 4.88 & juvenile & CB, McKewn \\
\hline CCNHM 1572 & $\sim 22$ & $\sim 34$ & $\mathrm{~A} 1-\mathrm{A} 3, \mathrm{a} 1-\mathrm{a} 3$ & 4.84 & juvenile & CB, McKewn \\
\hline CCNHM 5174 & $\sim 18$ & 10 & L4 & 1.82 & neonate & CB, McKewn \\
\hline CCNHM 1576 & 35 & 30 & L4 & 5.35 & juvenile & CB, McKewn \\
\hline CCNHM 5177 & 35 & 33 & A2 & 3.97 & juvenile & CB, McKewn \\
\hline CCNHM 5178 & $\sim 38$ & $\sim 40$ & a1-a2 & 5.62 & juvenile & CB, McKewn \\
\hline CCNHM 5179 & 33 & 29 & L1 & 4.17 & juvenile & CB, McKewn \\
\hline CCNHM 5180 & $\sim 40$ & 34 & a1 & 4.98 & juvenile & CB, McKewn \\
\hline CCNHM 5181 & 38 & 22 & L2 & 3 & juvenile & CB, McKewn \\
\hline CCNHM 5182 & $\sim 28$ & 23 & L1 & 3.32 & juvenile & CB, McKewn \\
\hline CCNHM 5183 & 25 & 26 & $|1-| 2$ & 4.82 & juvenile & CB, McKewn \\
\hline CCNHM 5184 & $\sim 30$ & $\sim 25$ & L1 & 3.61 & juvenile & CB, McKewn \\
\hline CCNHM 5185 & $\sim 28$ & $\sim 19$ & L5 & 4.96 & juvenile & CB, McKewn \\
\hline CCNHM 5186 & $\sim 30$ & $\sim 29$ & a1 & 4.24 & juvenile & CB, McKewn \\
\hline CHM PV 541 & 47 & 48 & A1-a1 & 6.33 & juvenile & $\mathrm{CB}$, excavation \\
\hline CHM PV 21 & 46 & $\sim 41$ & L1-I1 & 6.56 & juvenile & $\mathrm{CB}$, excavation \\
\hline
\end{tabular}


TABLE 1 (continued).

\begin{tabular}{|c|c|c|c|c|c|c|}
\hline Specimen & $\mathrm{CW}(\mathrm{mm})$ & $\mathrm{CH}(\mathrm{mm})$ & Tooth Position & $\mathrm{TL}(\mathrm{m})$ & Age class & Formation \\
\hline CHM PV 22 & $\sim 33$ & $\sim 35$ & A2-a2 & 4.45 & juvenile & CB, excavation \\
\hline CHM PV 23 & 34 & 30 & L1 & 4.32 & juvenile & CB, excavation \\
\hline CHM PV 24 & 42 & $\sim 35$ & L1-L3 & 4.96 & juvenile & CB, excavation \\
\hline CHM PV 25 & 31 & 24 & $|1-| 2$ & 4.45 & juvenile & $\mathrm{CB}$, excavation \\
\hline CHM PV 26 & 37 & $\sim 30$ & L1 & 4.32 & juvenile & $\mathrm{CB}$, excavation \\
\hline CHM PV 27 & 33 & 28 & a1-a3 & 4.22 & juvenile & CB, excavation \\
\hline CHM PV 28 & 39 & 15 & A1-a1 & 1.97 & neonate & $\mathrm{CB}$, excavation \\
\hline CHM PV 281 & 46 & 40 & L1-L3, I1-I3 & 7.02 & adult & $\mathrm{CB}$, excavation \\
\hline CHM PV 29 & 41.2 & 31.1 & L3-I3 & 6.02 & juvenile & $\mathrm{CB}$, excavation \\
\hline CHM PV 297 & 26.4 & 22.2 & L1-L3, I1-I3 & 3.94 & juvenile & CB, excavation \\
\hline CHM PV 298 & $\sim 32$ & $\sim 41$ & A1-a1 & 5.4 & juvenile & $\mathrm{CB}$, excavation \\
\hline CHM PV 30 & 35 & 41 & A1-a1 & 5.4 & juvenile & $\mathrm{CB}$, excavation \\
\hline CHM PV 31 & 33 & $\sim 42$ & $\mathrm{~A} 1-\mathrm{a} 1$ & 5.53 & juvenile & $\mathrm{CB}$, excavation \\
\hline CHM PV 32 & 28 & 26 & A1-A3, a1-a3 & 3.7 & juvenile & $\mathrm{CB}$, excavation \\
\hline CHM PV 33 & $\sim 35$ & $\sim 36$ & A1-A3, a1-a3 & 5.12 & juvenile & $\mathrm{CB}$, excavation \\
\hline CHM PV 34 & 36 & 34 & a1 & 4.98 & juvenile & CB, excavation \\
\hline CHM PV 35 & 40 & $\sim 37$ & L1 & 5.31 & juvenile & $\mathrm{CB}$, excavation \\
\hline CHM PV 36 & 32 & $\sim 38$ & a1 & 5.58 & juvenile & CB, excavation \\
\hline CHM PV 37 & 34 & $\sim 40$ & a1 & 5.88 & juvenile & $\mathrm{CB}$, excavation \\
\hline CHM PV 38 & 33 & 27 & L1-L3, I1-I3 & 4.77 & juvenile & $\mathrm{CB}$, excavation \\
\hline CHM PV 39 & 42 & $\sim 26$ & L3-I3 & 5.01 & juvenile & CB, excavation \\
\hline CHM PV 40 & $\sim 27$ & 24 & $11-12$ & 4.45 & juvenile & $\mathrm{CB}$, excavation \\
\hline CHM PV 41 & $\sim 30$ & $\sim 32$ & $\mathrm{~A} 1-\mathrm{a} 1$ & 4.21 & juvenile & $\mathrm{CB}$, excavation \\
\hline CHM PV 42 & 36 & $\sim 37$ & A1-A3, a1-a3 & 5.26 & juvenile & CB, excavation \\
\hline CHM PV 43 & 37 & $\sim 33$ & L1-L2, I1-I2 & 5.36 & juvenile & $\mathrm{CB}$, excavation \\
\hline CHM PV 44 & 36 & $\sim 40$ & A1-A3, a1-a3 & 5.69 & juvenile & $\mathrm{CB}$, excavation \\
\hline CHM PV 45 & 44 & 35 & L3 & 5.1 & juvenile & CB, excavation \\
\hline CHM PV 46 & 29 & $\sim 27$ & L1-I1 & 4.35 & juvenile & $\mathrm{CB}$, excavation \\
\hline CHM PV 47 & 31 & $\sim 24$ & L1-L2, I1-I2 & 3.91 & juvenile & $\mathrm{CB}$, excavation \\
\hline CHM PV 48 & $\sim 34$ & 27.8 & L2-L4 & 4.27 & juvenile & CB, excavation \\
\hline CHM PV 49 & 45 & $\sim 33$ & L1-L2 & 4.61 & juvenile & $\mathrm{CB}$, excavation \\
\hline CHM PV 50 & 34.9 & $\sim 29$ & L1-L3, I1-I3 & 5.11 & juvenile & $\mathrm{CB}$, excavation \\
\hline CHM PV 51 & 34 & 32 & L1 & 4.6 & juvenile & CB, excavation \\
\hline CHM PV 52 & 34 & $\sim 31$ & L1-L3 & 4.4 & juvenile & $\mathrm{CB}$, excavation \\
\hline CHM PV 53 & $\sim 34$ & $\sim 30$ & L2-L3 & 4.22 & juvenile & $\mathrm{CB}$, excavation \\
\hline CHM PV 54 & $\sim 33$ & $\sim 31$ & A3-a3 & 5.21 & juvenile & $\mathrm{CB}$, excavation \\
\hline CHM PV 540 & 83.6 & 74.5 & A2-A3,a2-a3 & 10.88 & adult & $\mathrm{CB}$, excavation \\
\hline CHM PV 55 & 44 & $\sim 32$ & L1-L2 & 4.47 & juvenile & $\mathrm{CB}$, excavation \\
\hline CHM PV 56 & $\sim 44$ & $\sim 32$ & a3 & 5.53 & juvenile & CB, excavation \\
\hline CHM PV 564 & 38.2 & 32.3 & L1-L3 & 4.58 & juvenile & $\mathrm{CB}$, excavation \\
\hline CHM PV 57 & $\sim 27$ & $\sim 20$ & L4-14 & 5.06 & juvenile & $\mathrm{CB}$, excavation \\
\hline CHM PV 58 & $\sim 35$ & 32 & L1-L2, I1-I2 & 5.2 & juvenile & CB, excavation \\
\hline CHM PV 59 & 35 & $\sim 30$ & L3 & 4.37 & juvenile & $\mathrm{CB}$, excavation \\
\hline CHM PV 60 & 30 & 29 & A1-a1 & 3.82 & juvenile & $\mathrm{CB}$, excavation \\
\hline
\end{tabular}


TABLE 1 (continued).

\begin{tabular}{|c|c|c|c|c|c|c|}
\hline Specimen & $\mathrm{CW}(\mathrm{mm})$ & $\mathrm{CH}(\mathrm{mm})$ & Tooth Position & $\mathrm{TL}(\mathrm{m})$ & Age class & Formation \\
\hline CHM PV 61 & $\sim 30$ & 29 & a1 & 4.24 & juvenile & $\mathrm{CB}$, excavation \\
\hline CHM PV 62 & $\sim 32$ & 24 & L1-L3 & 3.41 & juvenile & $\mathrm{CB}$, excavation \\
\hline CHM PV 63 & 36 & $\sim 40$ & a1-a3 & 6.06 & juvenile & $\mathrm{CB}$, excavation \\
\hline CHM PV 64 & $\sim 48$ & 33 & $\mid 1$ & 5.89 & juvenile & $\mathrm{CB}$, excavation \\
\hline CHM PV 65 & $\sim 34$ & 41 & a1 & 6.03 & juvenile & $\mathrm{CB}$, excavation \\
\hline CHM PV 66 & $\sim 42$ & $\sim 34$ & 12 & 6.54 & juvenile & $\mathrm{CB}$, excavation \\
\hline CHM PV 67 & 25 & 21 & 14 & 6.88 & adult & $\mathrm{CB}$, excavation \\
\hline CHM PV 68 & 38 & $\sim 30$ & L1-L3 & 4.25 & juvenile & $\mathrm{CB}$, excavation \\
\hline CHM PV 69 & $\sim 48$ & 34.4 & a1 & 5.04 & juvenile & $\mathrm{CB}$, excavation \\
\hline CHM PV 70 & 25.1 & 26.8 & A3 & 4.41 & juvenile & $\mathrm{CB}$, excavation \\
\hline CHM PV 71 & $\sim 27$ & $\sim 25$ & L1-I1 & 4.03 & juvenile & $\mathrm{CB}$, excavation \\
\hline CHM PV 72 & $\sim 27$ & $\sim 31$ & L4-L5 & 6.83 & adult & $\mathrm{CB}$, excavation \\
\hline CHM PV 73 & $\sim 33$ & $\sim 30$ & a1-a2 & 4.2 & juvenile & $\mathrm{CB}$, excavation \\
\hline CHM PV 75 & 30.6 & 31.3 & L2-L4 & 4.8 & juvenile & $\mathrm{CB}$, excavation \\
\hline CHM PV 76 & 29.8 & $\sim 25$ & L1-I1 & 4.03 & juvenile & $\mathrm{CB}$, excavation \\
\hline CHM PV 77 & 29 & 20 & L4-L5 & 4.4 & juvenile & $\mathrm{CB}$, excavation \\
\hline CHM PV 78 & $\sim 35$ & 35 & A1-a1 & 4.61 & juvenile & CB, excavation \\
\hline CHM PV 79 & $\sim 34$ & 26 & L1-L2 & 3.64 & juvenile & $\mathrm{CB}$, excavation \\
\hline CHM PV 80 & 39 & 35.5 & L1-L3, I1-I3 & 6.24 & juvenile & $\mathrm{CB}$, excavation \\
\hline CHM PV 81 & 24.2 & $\sim 23$ & L1-L3, I1-I3 & 4.07 & juvenile & CB, excavation \\
\hline CHM PV 82 & $\sim 26$ & 22.3 & L1-L3, I1-I3 & 3.95 & juvenile & $\mathrm{CB}$, excavation \\
\hline CHM PV 83 & $\sim 27$ & 15 & L1-L3 & 2.15 & neonate & $\mathrm{CB}$, excavation \\
\hline CHM PV 84 & $\sim 20$ & 18.1 & L1-L2, I1-I2 & 2.96 & juvenile & CB, excavation \\
\hline CHM PV 86 & 27 & $\sim 24$ & A1-A3, a1-a3 & 3.41 & juvenile & $\mathrm{CB}$, excavation \\
\hline CHM PV 87 & 30 & $\sim 30$ & A2-A3, a2-a3 & 4.42 & juvenile & $\mathrm{CB}$, excavation \\
\hline CHM PV 88 & $\sim 16$ & $\sim 13$ & 12 & 2.51 & neonate & $\mathrm{CB}$, excavation \\
\hline CHM PV 89 & $\sim 25$ & $\sim 17$ & L1-L3, I1-I3 & 3.03 & juvenile & $\mathrm{CB}$, excavation \\
\hline CHM PV 90 & 26.2 & $\sim 20$ & L1-L3, I1-I3 & 3.55 & juvenile & $\mathrm{CB}$, excavation \\
\hline CHM PV 91 & $\sim 37$ & $\sim 28$ & L1-L2 & 3.92 & juvenile & CB, excavation \\
\hline CHM PV 9835 & 31.8 & 28.2 & L1-I1 & 4.54 & juvenile & $\mathrm{CB}$, excavation \\
\hline CHM PV 9836 & 24.9 & 21.6 & L1-L3 & 3.07 & juvenile & $\mathrm{CB}$, excavation \\
\hline CHM PV 9837 & 34.7 & $\sim 31$ & L1-L3, I1-I3 & 5.46 & juvenile & $\mathrm{CB}$, excavation \\
\hline CHM PV 9838 & $\sim 32$ & $\sim 26$ & a1-I1 & 4.09 & juvenile & $\mathrm{CB}$, excavation \\
\hline CHM PV 9839 & $\sim 78$ & $\sim 74$ & a1 & 10.94 & adult & $\mathrm{CB}$, excavation \\
\hline CHM PV 9840 & $\sim 27$ & $\sim 22$ & L5 & 5.75 & juvenile & $\mathrm{CB}$, excavation \\
\hline CHM PV 9842 & 28.6 & 29.3 & L4 & 5.23 & juvenile & CB, excavation \\
\hline CHM PV 9843 & 28.7 & $\sim 27$ & A1-A3, a1-a3 & 3.84 & juvenile & $\mathrm{CB}$, excavation \\
\hline CHM PV 9844 & 28.6 & $\sim 22$ & 12 & 4.24 & juvenile & CB, excavation \\
\hline CHM PV 9845 & 29.5 & $\sim 23$ & L1 & 3.32 & juvenile & CB, excavation \\
\hline CHM PV 9846 & $\sim 32$ & $\sim 25$ & L4 & 4.47 & juvenile & $\mathrm{CB}$, excavation \\
\hline CHM PV 9847 & 30.9 & 34.3 & A1-a1 & 4.52 & juvenile & CB, excavation \\
\hline CHM PV 9848 & - & 40.7 & L2 & 5.52 & juvenile & $\mathrm{CB}$, excavation \\
\hline CHM PV 9849 & 31.8 & $\sim 37$ & A1-a1 & 4.87 & juvenile & $\mathrm{CB}$, excavation \\
\hline CHM PV 9850 & 25.2 & $\sim 23$ & $\mathrm{~A} 1$ & 2.7 & juvenile & CB, excavation \\
\hline
\end{tabular}


TABLE 1 (continued).

\begin{tabular}{lccccll}
\hline Specimen & CW(mm) & CH(mm) & Tooth Position & TL(m) & Age class & Formation \\
\hline CHM PV 9851 & 24 & $\sim 22$ & A2 & 2.64 & juvenile & CB, excavation \\
CHM PV 9852 & 33.8 & 33.5 & A2 & 4.03 & juvenile & CB, excavation \\
CHM PV 9853 & 41.5 & 35 & I2 & 6.73 & juvenile & CB, excavation \\
CHM PV 9855 & $\sim 37$ & $\sim 34$ & A2 & 4.1 & juvenile & CB, excavation \\
CHM PV 9856 & $\sim 34$ & 29.1 & I3 & 7 & adult & CB, excavation \\
CCNHM 5994 & 76.3 & 81.4 & A1 & 9.44 & adult & Ex situ \\
PV-7271 & 87.01 & $\sim 76.90$ & A3-L2 & 7.75 & adult & Ex situ \\
PV-7281 & 74.99 & $\sim 97$ & A1 & 11.22 & adult & Ex situ \\
PV-7269 & $\sim 83.20$ & $\sim 84.93$ & L1-L3 & 9.83 & adult & Ex situ \\
PV-7279 & $\sim 75.90$ & $\sim 92$ & A1-A2 & 10.88 & adult & Ex situ \\
PV-7260 & $\sim 83.26$ & $\sim 78.34$ & A1 & 9.07 & adult & Ex situ \\
\hline
\end{tabular}

gists who collected all vertebrate fossils as a 'census assemblage' at two different localities, decades apart. At the Chandler Bridge excavation site, a controlled excavation of an unusually densely fossiliferous bonebed $\left(550 \mathrm{~m}^{2}\right.$; Sanders, 1980) within bed 3 of the Chandler Bridge Formation was performed over six weeks in 1970, seven weeks in 1971, and seven weeks in summer 1972 (Sanders, 1980). Because all specimens of Carcharocles angustidens from this excavation site were sampled, collection bias is not a factor. At the McKewn Subdivision locality, teeth were collected by one of us (R.W. Boessenecker, S.J. Boessenecker, and A. Gale) over three months while the locality was inaccessible to hobby collectors. Rather than being collected under controlled quarrying operations like the Chandler Bridge excavation site, these specimens were naturally weathered out prior to collection from a somewhat larger area of outcrop $\left(907 \mathrm{~m}^{2}\right.$; measured in ImageJ based upon photogrammetric models of the locality) - similarly not biased by hobby collecting, but collected intermittently and aided by natural weathering rather than systematic quarrying. Because all vertebrate fossils from the McKewn locality were collected, collection bias can be excluded as a factor in interpreting the size distribution of these teeth. We also evaluated the taphonomic condition of specimens, examining them for evidence of reworking that could potentially bias the results, including evidence of abrasion, fragmentation, bioerosion, and phosphatization.

Tooth shape and size vary within Carcharocles angustidens as in other sharks. If the tooth position is incorrect, body length estimates might be overestimated. In order to exclude tooth posi- tion as a variable, each tooth was identified to approximate position based on comparison with the only known dentition of $C$. angustidens (OU 22261, Gottfried and Fordyce, 2001), but use A3 over I1 (intermediate) and include the posteriors as a continuation of the laterals after Shimada (2002) and Perez et al. (2021). Anterior teeth are the largest, and generally have the longest (relative to root width) and most symmetrical crowns; lateral teeth are relatively smaller than anteriors and have proportionally shorter and more distally inclined crowns, and increasingly have proportionally smaller crowns in further distal tooth positions closer to the commissure (=posteriors of some authors, e.g., Gottfried and Fordyce, 2001). Because individual specimens could not always be confidently placed to a single position, equations for multiple adjacent (and plausible) positions were calculated, and an average of these was used and entered into Table 1.

\section{Taxonomic Note}

In recent years the taxonomy of Paleogene megatoothed sharks has grown considerably. The transition from Otodus obliquus to serrated Carcharocles angustidens has now been subdivided into a number of different chronospecies of uncertain utility, largely by Zhelezko and Kozlov (1999), either by the introduction of newly named taxa or resurrection of disused taxa, including (but not limited to) the Eocene species Otodus aksuaticus, Otodus minor, Otodus naidini, Otodus poseidoni, Otodus sokolovi, and several subspecies of these. Some of these have been subdivided or included into subgenera (Cappetta, 2012), which are generally not advised in vertebrate paleontol- 
ogy (Ehret and Ebersole, 2014). Most of these species in the transition from non-serrated $O$. obliquus to fully serrated $C$. angustidens are differentiated entirely on the basis of slight variations in cusplet shape and crown proportions (broad, narrow). Because large sample sizes reveal considerable variation in cusplet morphology (see below), we consider the late Eocene $C$. sokolovi to be a junior synonym of Carcharocles auriculatus as the cusplet morphology of these broadly overlaps in the context of variation within single individuals documented by Gottfried and Fordyce (2001). Most of these species and subspecies are subjectively diagnosed (Ehret and Ebersole, 2014), and variation within these species is not addressed or quantified (Kent, 2018). Despite careful stratigraphic work by Zhelezko and Kozlov (1999), morphometric studies within a stratigraphic context have not been attempted to evaluate the diagnoseability of these taxa (but see King et al., 2013; Perez et al., 2019), and we note that King et al. (2013) identified only a single transitional species - Carcharocles aksuaticus -- between nonserrated Otodus obliquus and fully serrated Carcharocles auriculatus. We follow Kent (2018) and Perez et al. (2019) in using $C$. chubutensis over $C$. subauriculatus owing to the lack of cusplets in and locality provenience for the $C$. subauriculatus type specimen (but see Purdy et al., 2001). Further evaluation will require morphometric study of statistically large samples within a detailed stratigraphic context (e.g., Perez et al., 2019).

The genus-level taxonomy of megatoothed sharks has recently changed somewhat, but generally stabilized around either the use of Carcharocles or Otodus (Shimada et al., 2017, and references therein). Shimada et al. (2017) proposed placing all species of otodontids with serrated teeth (Carcharocles auriculatus, Carcharocles angustidens, Carcharocles chubutensis, Carcharocles megalodon) into the genus Otodus in order to prevent the genus Otodus from being para- or polyphyletic, and because Otodus has priority over Carcharocles. The cladograms presented in Shimada et al. (2017) are hypothetical (Shimada et al., 2017: figs. 3-4) and do not represent trees derived from cladistic analyses of morphological data. Though subsequent studies have followed this taxonomic decision (e.g., Boessenecker et al., 2019; Cooper et al., 2020; Herraiz et al., 2020; Perez et al., 2021), not all have (e.g., Godfrey et al., 2018; Kent, 2018; Perez et al., 2019). An exhaustive review of this subject is provided by Kent (2018: 80-85). Because ancestor-descendant relationships in this group have not been demonstrated (or evaluated) quantitatively (e.g., cladistics, stratophenetics), and because taxonomic revision of Otodus and Eocene Carcharocles spp. is still needed (Ehret and Ebersole, 2014; Kent, 2018), we agree with Kent (2018) and conservatively suggest that use of Carcharocles is still acceptable for the time being; we further echo Kent's (2018) call for further research on early megatoothed sharks and emphasize the need for application of quantitative and/or cladistic methods.

\section{RESULTS}

New specimens $(n=136)$ from Ashley and Chandler Bridge Formation exposures at the McKewn subdivision and Chandler Bridge excavation site (Figures 3-5) are assignable to Carcharocles angustidens because they possess regular serrations (rather than the more irregular serrations of Eocene Carcharocles auriculatus), triangular lateral cusplets with regular fine serrations separated from the main cusp by a distinct notch, U-shaped roots, rectangular to triangular root lobe apices, and relatively narrow crowns in comparison to geochronologically younger members of this lineage (e.g., Carcharocles chubutensis, Carcharocles megalodon). Just over half $(n=73)$ represent lateral tooth positions, and just under half $(n=54)$ represent anterior tooth positions.

Maximum estimated body length of Carcharocles angustidens appears to be in the range of 10$11 \mathrm{~m}$ (Figure 2, Table 1). Many of these large specimens were collected ex-situ, and some are damaged with estimated crown lengths. The largest individual specimen was ChM PV 7281, which is incomplete, but with an estimated body length of 11.2 meters. The largest specimen with a completely preserved crown is CCNHM 5994, a slightly smaller A1 with an estimated body length of 9.44 $\mathrm{m}$. Other specimens slightly smaller than this represented estimated body lengths of 7.75-10.88 m (Figure 2, Table 1), suggestive of a maximum body length between 11 and $12 \mathrm{~m}$, broadly in accordance with Shimada et al. (2020).

Crown height measurements of Carcharocles angustidens teeth from the Chandler Bridge Formation exposed in the McKewn Subdivision (CCNHM collections), and Chandler Bridge excavation site (ChM collections) range from $10 \mathrm{~mm}$ to $74.5 \mathrm{~mm}$ (Table 1). The McKewn Subdivision sample consists of rare neonates $(3.12 \%, n=1)$ and completely lacks adults $(0 \%, \mathrm{n}=0)$, and is dominated by juveniles $(96.87 \%, n=31$; Figure $6 \mathrm{~A}$; Table 

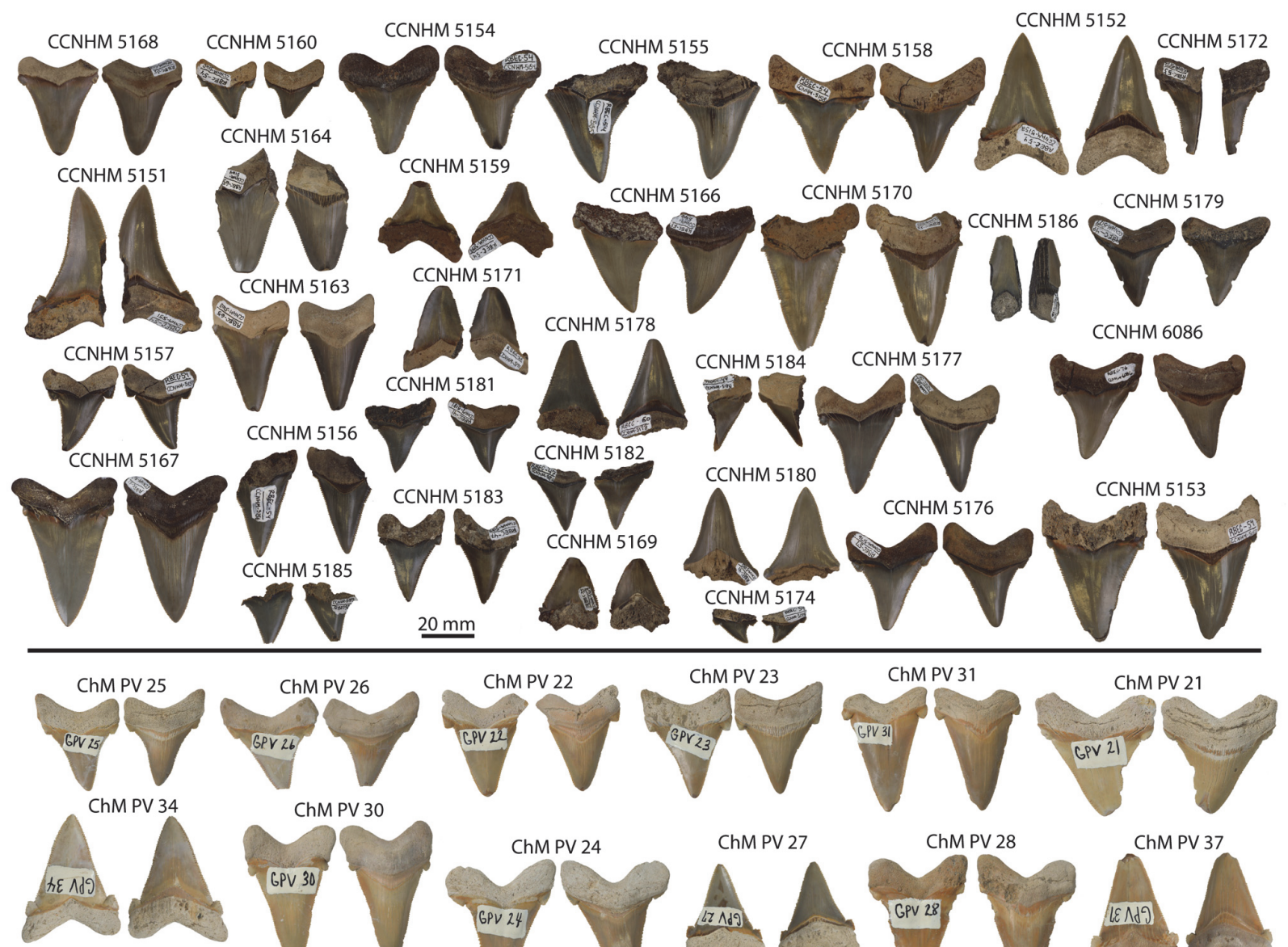

ChM PV 30
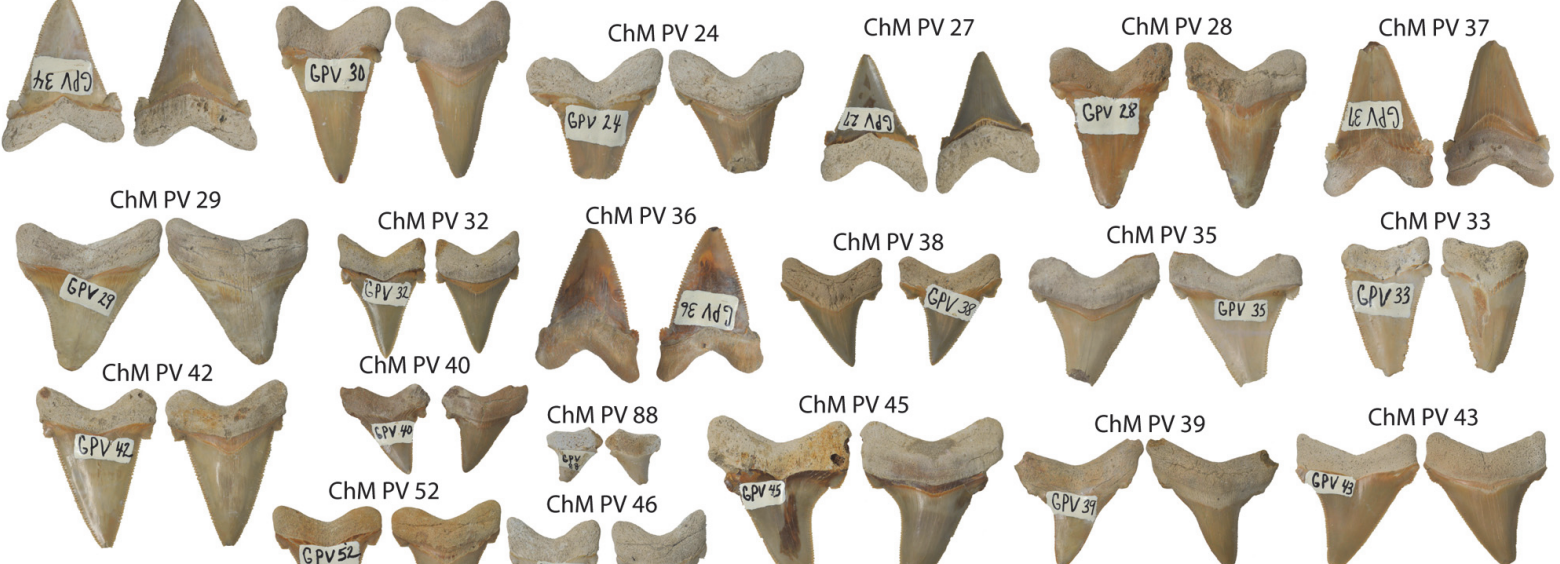

ChM PV 33
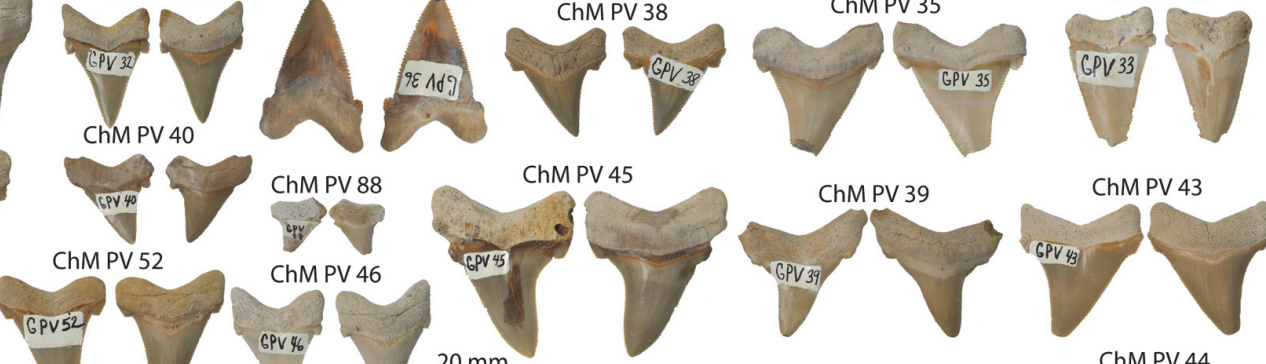

ChM PV 49
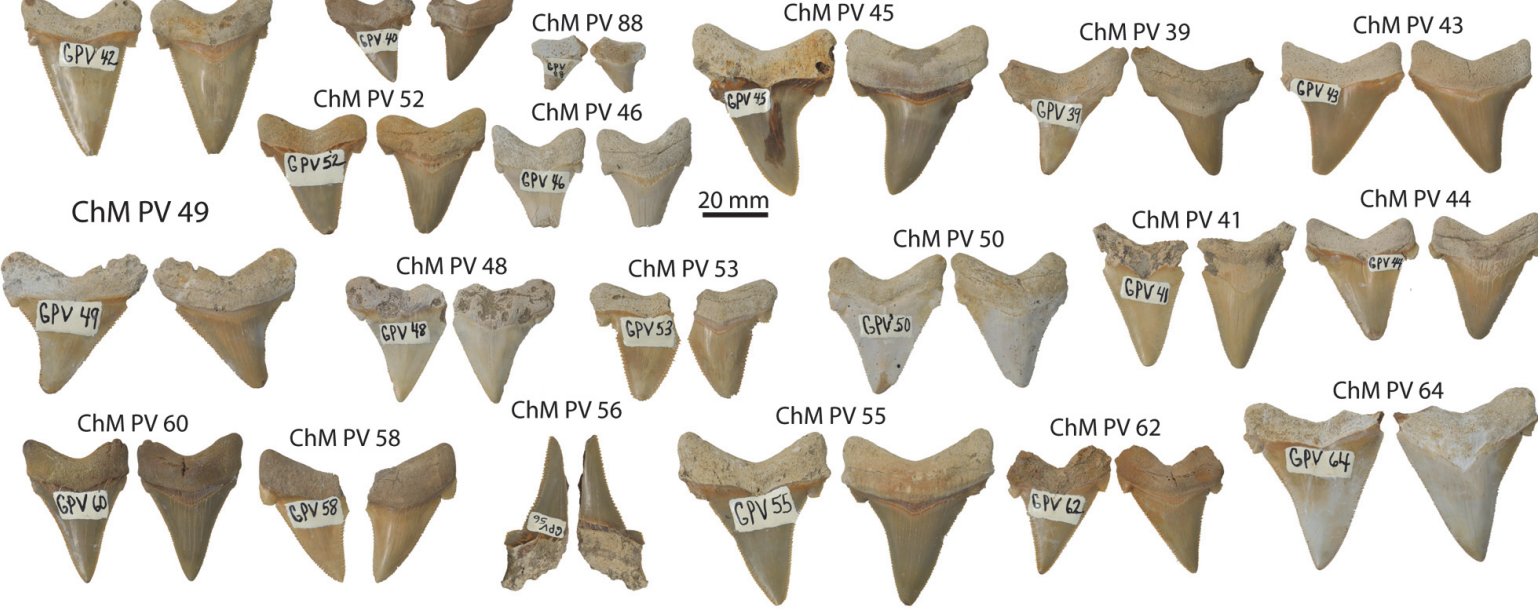

ChM PV 44
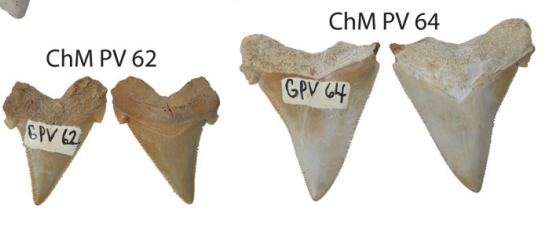

FIGURE 3. Teeth of Carcharocles angustidens from the Chandler Bridge Formation (late Oligocene), from the McKewn subdivision sample (top, CCNHM specimens) and part of the Chandler Bridge excavation sample (bottom, ChM specimens). Teeth shown in anatomical orientation (e.g., crown downwards for upper teeth); labial on left, lingual on right in all cases. All specimens shown to scale. 


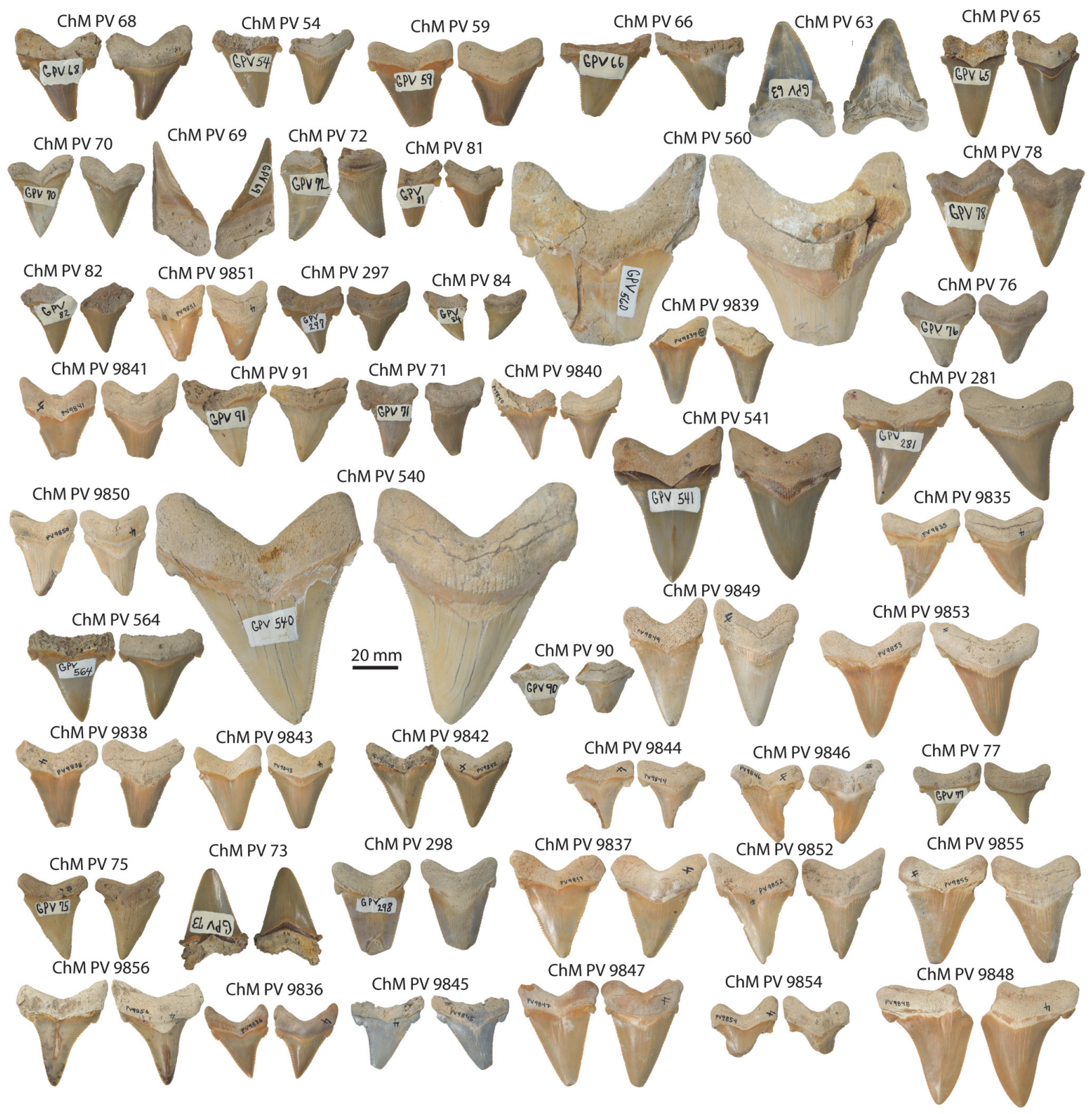

FIGURE 4. Teeth of Carcharocles angustidens from the Chandler Bridge Formation (late Oligocene), from the Chandler Bridge excavation sample, in anatomical orientation (e.g., crown downwards for upper teeth); labial on left, lingual on right in all cases. All specimens shown to scale.

1). The Chandler Bridge excavation sample is similarly dominated by juveniles $(90.52 \%, \mathrm{n}=86)$, with rare neonates $(3.15 \%, n=3)$ and adults $(6.31 \%$, $\mathrm{n}=6$; Figure 6B; Table 1). The combined assemblage is therefore also dominated by juveniles $(92.1 \%, n=117)$, with few neonates $(3.14 \%, n=4)$ and adults $(4.72 \%, \mathrm{n}=6$; Figure $6 \mathrm{C}$; Table 1$)$.

The majority of teeth from these samples have a crown height around $30 \mathrm{~mm}$ (median crown height: $30 \mathrm{~mm}$; mean crown height: $30.7 \mathrm{~mm}$; Table
1). Using published formulae for calculating body length of lamniform sharks (Shimada, 2002), the majority of Carcharocles angustidens from the combined sample were reconstructed between 4-6 $\mathrm{m}$ long ( $\mathrm{n}=83$; 65.35\%; Figure 6C; Table 1). The McKewn subdivision and Chandler Bridge excavation collections do not seriously differ from one another.

The estimated body length of the largest specimens from the McKewn sample ranges to 7 

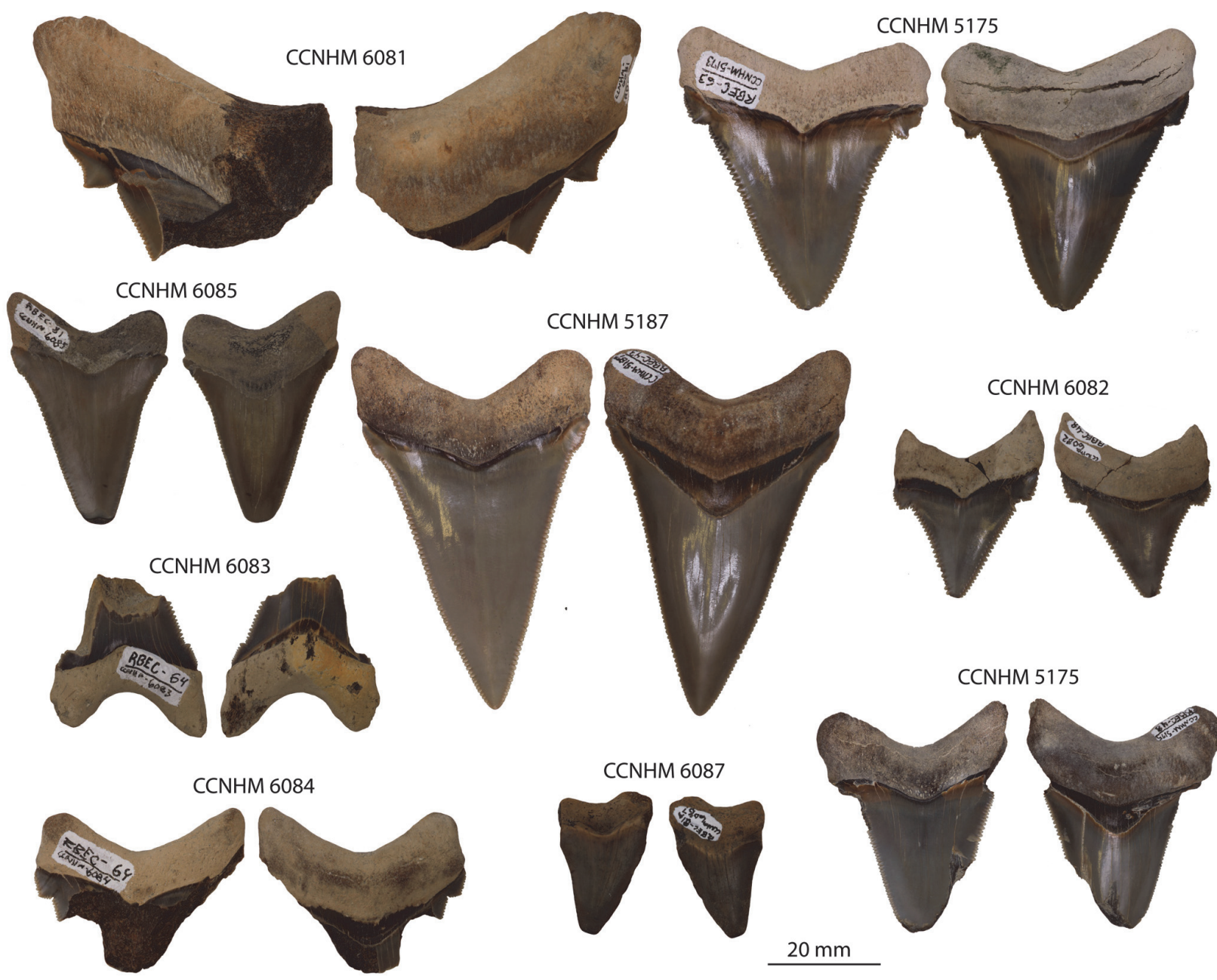

FIGURE 5. Teeth of Carcharocles angustidens from the Ashley Formation (early Oligocene), from the McKewn subdivision sample, in anatomical orientation (e.g., crown downwards for upper teeth); labial on left, lingual on right in all cases. All specimens shown to scale.

$\mathrm{m}$, whereas two outlier specimens occur within the Chandler Bridge excavation sample (Figures 4, 5B, Table 1): ChM PV 540 and 9839, each with estimated body lengths of 10.88 and $10.94 \mathrm{~m}$ (respectively), and similar in size to the largest known specimens of Carcharocles angustidens (Figures 2, 5B, Table 1).

A small sample of Carcharocles angustidens teeth from the Ashley Formation ( $n=9$; Figure 5) mostly represent juvenile specimens (CCNHM $5173,5187,6082,6085,6087$, and probably 5175 , $6083,6084)$. A single fragment of a large tooth (CCNHM 6081; Figure 5) must have had about a $70 \mathrm{~mm}$ crown width and rivals the two largest teeth from the Chandler Bridge excavation, ChM PV 540 and 9839 , likely representing $a+10 \mathrm{~m}$ long shark.

Some teeth are incomplete (e.g., CCNHM 5151, 5153, 5159, 5171, 5186, ChM PV 24, 46, 56,
69, 90, 298, 9854), some bear bioeroded roots (e.g., CCNHM 5153, 5156, 5159, ChM PV 41, 54, 73, 91, 9840), and some are lightly abraded (e.g., CCNHM 5179, 5180, 5186, ChM PV 29, 48, 63, 69, $75,76)$; most are not phosphatized and are remarkably uniform in coloration.

\section{DISCUSSION}

\section{Identification}

Otodontid teeth have been reported ex situ from various Oligocene-Pliocene strata in South Carolina, but few specimens have been reported from in situ Oligocene rocks. Purdy (1996) figured three teeth of "Carcharodon sp." from the Chandler Bridge Formation (USNM 476833, figure 3c of Purdy, 1996; ChM PV 540, and probably ChM PV 9849, figure $8 \mathrm{~b}$ and $8 \mathrm{a}$, respectively, of Purdy, 

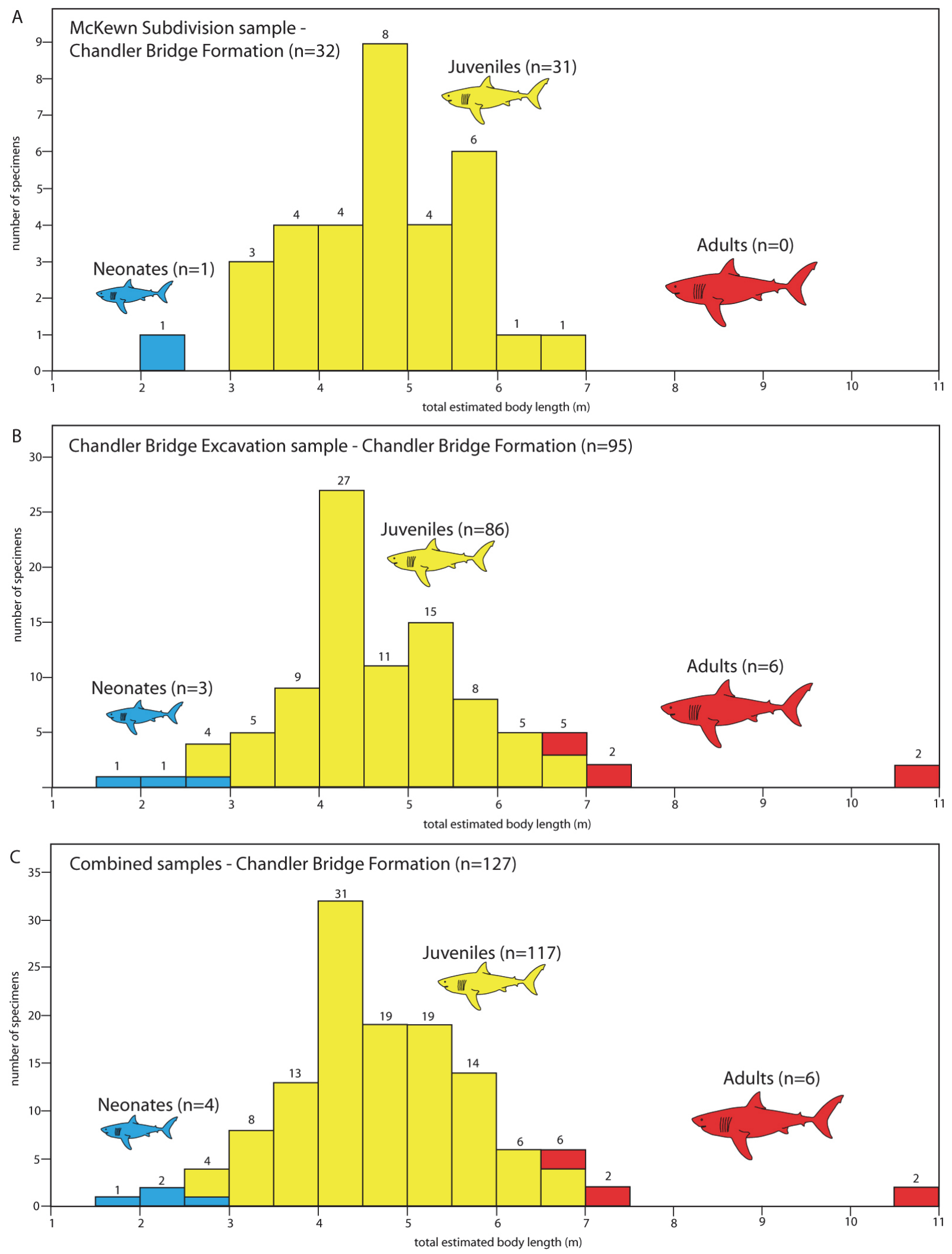

FIGURE 6. Histogram showing range of body length estimates from A) the McKewn subdivision sample $(n=32), B)$ the Chandler Bridge excavation sample ( $n=95)$, and $C$ ) combined samples from the Chandler Bridge Formation (McKewn subdivision and Chandler Bridge excavation; $n=127$ ). 
1996). Purdy (1996) cautiously refrained from identifying these to the species level. A single small tooth described by Cicimurri and Knight (2009) from the Chandler Bridge Formation was cautiously identified as Carcharocles sp. owing to incomplete separation of the cusplets from the crown. Several teeth from each sample similarly have an incomplete notch between the crown and the cusplet, more closely resembling Carcharocles chubutensis than Carcharocles angustidens. However, we consider this a case of morphological variation within a sample, and owing to 1) more specimens possessing notches than not $(66.6 \%$ of the McKewn subdivision sample, and $87 \%$ of the Chandler Bridge excavation sample; Figures 3 and $4), 2$ ) other features distinguishing these teeth from C. chubutensis (e.g., narrower crowns), and 3) Oligocene age (since $C$. chubutensis is an early Miocene chronospecies; Perez et al., 2019), we refer all serrated otodontid teeth from the Ashley and Chandler Bridge formations to $C$. angustidens. We further highlight morphological variation even within a single dentition of $C$. angustidens, represented by OU 22261 from the Oligocene of New Zealand, which preserves teeth with triangular cusplets separated by a distinct notch, triangular cusplets lacking a notch, and low, ridge-like cusplets (Gottfried and Fordyce, 2001: figures 3-4). Assemblage-level and dentition-level polymorphisms argue for caution when interpreting individual otodontid specimens and highlight the utility of large sample sizes with stratigraphic control. These results further cast doubt on supposedly diagnostic but in actuality subtle and variable features (Gottfried and Fordyce, 2001; Kent, 2018; this study) used to erect the overly-parsed taxonomy of Eocene otodontids.

\section{Nursery Area for Carcharocles angustidens}

Two newly reported census assemblages of Carcharocles angustidens from the Chandler Bridge Formation are dominated by teeth of juveniles and neonates (Figures 3-5). Taphonomic bias in the form of size-sorting is unlikely to be a controlling factor because the bonebeds within the Chandler Bridge Formation at the McKewn subdivision locality include relatively small, delicate shark teeth ( $<0.5 \mathrm{~cm}$ diameter) and fish bones along with larger teeth, bones, and phosphate nodules (10-40 $\mathrm{cm})$. At the McKewn Subdivision locality, most vertebrates originated from one of two matrix supported bonebeds with a wide variety of bioclast sizes (teeth and fish bones under $1 \mathrm{~cm}$, and bones over $15 \mathrm{~cm}$ in length) and phosphate nod- ules in excess of $20 \mathrm{~cm}$ diameter. Owing to gradational lower and upper contacts, these are likely hiatal bonebeds rather than completely erosional in origin (Boessenecker et al., 2014). This evidence does not support size-sorting to have occurred during deposition. Collection bias also cannot be considered owing to controlled collecting methods and an absence of interference by hobby collectors (see above). Controlled excavation at the Chandler Bridge excavation further eliminates collection bias as a possibility. Despite lack of a formal taphonomic analysis, this assemblage has been prematurely interpreted as a stranding owing to the number of cetacean skeletons, variety of species, and apparent parallel development of skeletal material in rows aligned northwest/southeast (Erickson, 1990). However, it is also possible that this assemblage is a hiatal concentration like the majority of shallow marine bonebeds (e.g., Boessenecker et al., 2014). Tooth fracturing in this sample is consistent with feeding damage (Becker and Chamberlain, 2012); in some cases, some damage in the McKewn sample was possibly caused by machinery or modern weathering (CCNHM 5151, 5164,5172 ) and other fracturing happened during collection (CCNHM 5157, 5158) or was likely exacerbated by bioerosion of the root (CCNHM 5155, $5170,5182,5185)$. Light abrasion is typical in parautochthonous shallow marine assemblages (Boessenecker et al., 2014), perhaps suggestive of a hiatal origin rather than a stranding as envisioned by Erickson (1990). Similarity in density and coloration suggests a lack of phosphatization, which, in concert with absence of strong abrasion (e.g., stage 2 of Boessenecker et al., 2014: fig. 5), point towards these teeth being autochthonous or at most parautochthonous, rather than allochthonous (reworked) in taphonomic origin. Lastly, Osedax bioerosion is unusually common in Oligocene shallow marine vertebrate assemblages worldwide relative to younger Neogene assemblages (see Boessenecker and Fordyce, 2015), but reported from shark teeth herein for the first time. A similar incidence of occasional light abrasion, somewhat more frequent Osedax bioerosion, and typical tooth fracturing is present in the McKewn Subdivision sample. Altogether, taphonomic aspects of the Chandler Bridge Formation samples of $C$. angustidens suggest autochthonous or, at worst, parautochthonous concentrations of fossils - without evidence of size-sorting or reworking. Because collection bias and taphonomic bias cannot reliably explain this size distribution, we interpret this 
assemblage as being genuinely dominated by neonatal and juvenile teeth.

Three criteria have been proposed to identify paleo-nursery areas for extinct sharks: nursery areas must consist of 1) relatively shallow water environments with 2 ) high primary productivity with abundant prey species, and 3) a preponderance of young individuals (Castro, 1993; Heithaus, 2007; Heupel et al., 2007; Pimiento et al., 2010; Landini et al., 2017; Villafaña et al., 2020; Herraiz et al., 2020). The Chandler Bridge Formation is clearly a shallow shelf deposit, and some evidence from this unit suggests the Charleston Embayment was somewhat protected (Katuna et al., 1997). Primary productivity was evidently high, with a rich marine vertebrate fossil assemblage (Sanders et al., 1982; Cicimurri and Knight, 2009; Boessenecker and Geisler, 2018, and references therein) and widespread phosphogenesis (Katuna et al., 1997) indicative of high primary productivity (Föllmi, 1996). Lastly, our combined collections are dominated by juveniles $(92.1 \%$ of the sample), and, with the exception of two outlier specimens from the Chandler Bridge excavation (ChM PV 540 and 9839), most of the largest teeth represent relatively large juveniles or small or adults in both samples. All three criteria are satisfied by the Chandler Bridge Formation. This study therefore confirms the hypothesis of Purdy (1996) that the Chandler Bridge Formation records a nursery area for Carcharocles angustidens in the Charleston Embayment.

A small sample of Carcharocles angustidens teeth is available from the Ashley Formation $(n=9$; Figure 5), and most (except for CCNHM 6081) are also within the juvenile size range (Table 1). The Ashley Formation represents somewhat deeper deposition but 1) has yielded a diverse marine vertebrate fauna similar to the Chandler Bridge Formation, 2) is also rich in phosphate indicating high primary productivity, and 3) was deposited within the same protected Charleston Embayment. At present, the limited sample suggests that the Charleston Embayment was a C. angustidens nursery area during deposition of the Ashley Formation. We hypothesize that this embayment was a nursery area for several million years, and predict that a larger sample from the Ashley Formation will bear this out. Most other specimens of $C$. angustidens collected in situ from other Ashley Formation localities are also from juveniles (e.g., CCNHM 1901). Critically, these are the first records of Carcharocles angustidens to be reported from the Ashley Formation (Figure 5), and indeed among the very first sharks ever reported from the unit. The Ashley Formation is not as richly fossiliferous as the overlying Chandler Bridge Formation, and discoveries from it have been more haphazard and biased towards cetaceans, sirenians, and sea turtles. Future studies of the McKewn subdivision assemblage will greatly expand knowledge of the marine vertebrate record from this unit.

Furthermore, it is noteworthy that nearly identical collections from the same strata were made in duplicate at two different (but nearby $-2.4 \mathrm{~km}$ separation) localities by different field paleontologists and decades apart from one another. These data demonstrate the power of careful technical fieldwork, and reciprocally enhance the juvenileweighted body size signal documented herein. We predict that future 'census' collections from the Ashley and Chandler Bridge formations will be similarly dominated by juvenile teeth. We further highlight the fact that similar modern embayments along the South Carolina coast are nursery areas for multiple shark species (Castro, 1993) and propose future similar studies of other commonly preserved sharks in the Oligocene of South Carolina, including Physogaleus, Galeocerdo, and Hemipristis, as juvenile teeth of these taxa are abundant in both Oligocene formations and may have also used the embayment as a nursery ground.

\section{CONCLUSIONS}

New assemblages of teeth of the extinct megatoothed shark Carcharocles angustidens from the Oligocene Chandler Bridge Formation of South Carolina are dominated by small teeth representing juveniles, and to a lesser extent, neonates. Owing to the shallow, warm, and somewhat protected geography of the Charleston Embayment, high primary productivity and abundant prey species, and a preponderance of small individuals, the Charleston Embayment is supported as a nursery area for extinct megatoothed sharks. The largest known teeth of $C$. angustidens indicate a maximum length of around 10-11 m. Fossil teeth representing the first known specimens of $C$. angustidens from the underlying Ashley Formation are also mostly small, perhaps indicating a nursery area throughout the Oligocene epoch. Modern species of Carcharhinus, Rhizoprionodon, Mustelus, and Sphyrna use nearby Bulls' Bay, South Carolina, as a nursery area today (Castro, 1993). Shark faunas have changed over time and the Charleston embayment may have filled with sediment, but new nurseries have been established in favorable habitats nearby, highlighting the suitability of the South 
Carolina coast for sharks over the recent geologic past.

\section{ACKNOWLEDGMENTS}

Thanks to K. Shimada and two anonymous reviewers for their constructive review comments, which greatly improved the quality of this paper. We thank the editor for comments and patience. Locality access was graciously provided by DR Horton Construction (McKewn Subdivision) and Trolley Enterprises (Chandler Bridge excavation); and thanks in particular to Bryan Doster and Ed Riley for facilitating field access (respectively). We thank Sarah J. Boessenecker and Ashby Gale for field assistance at the McKewn subdivision, and the hard work of $\mathrm{Al}$ Sanders and Charleston Museum volunteers at the Chandler Bridge excavation. Thanks to Sarah J. Boessenecker from the Mace Brown Museum of Natural History for access to collections, constructive comments, and moral support. We also thank Jessica Peragine from the Charleston Museum for her assistance. Thanks to Dana J. Ehret for discussions that improved the quality of this paper. Lastly, we begrudgingly thank whoever wrote "Baby shark".

\section{REFERENCES}

Abler, W.L. 1992. The serrated teeth of tyrannosaurid dinosaurs, and biting structures in other animals. Paleobiology, 18:161-183. https://doi.org/10.1017/S0094837300013956

Aguilera, O.A., García, L., and Cozzuol, M.A. 2008. Giant-toothed white sharks and cetacean trophic interaction from the Pliocene Caribbean Paraguaná Formation. Paläontologische Zeitschrift, 82:204-208. https://doi.org/10.1007/BF02988410

Becker, M.A. and Chamberlain, J.A. 2012. Squalicorax chips a tooth: a consequence of feedingrelated behavior from the lowermost Navesink Formation (Late Cretaceous: CampanianMaastrichtian) of Monmouth County, New Jersey, USA. Geosciences, 2:109-1279. https://doi.org/10.3390/geosciences2020109

Boessenecker, R.W., Perry, F.A., and Schmitt, J.G. 2014. Comparative taphonomy, taphofacies, and bonebeds of the Mio-Pliocene Purisima Formation, Central California: strong physical control on marine vertebrate preservation in shallow marine settings. PLoS ONE, 9:e91419. https://doi.org/10.1371/journal.pone.0091419

Boessenecker, R.W. and Fordyce, R.E. 2015. Trace fossil evidence of predation upon boneeating worms on a baleen whale skeleton from the Oligocene of New Zealand. Lethaia, 48(3):326-331. https://doi.org/10.1111/let.12108

Boessenecker, R.W. and Fordyce, R.E. 2017. A new eomysticetid from the Oligocene Kokoamu Greensand of New Zealand and a review of the Eomysticetidae (Mammalia, Cetacea). Journal of Systematic Palaeontology, 15(6):429-469. https://doi.org/10.1080/14772019.2016.1191045

Boessenecker, R.W. and Geisler, J.H. 2018. New records of the archaic dolphin Agorophius (Mammalia: Cetacea) from the upper Oligocene Chandler Bridge Formation of South Carolina, USA. PeerJ, 6:e5290. https://doi.org/10.7717/peerj.5290

Boessenecker, R.W., Ehret, D.J., Long, D.J., Churchill, M., Martin, E., and Boessenecker, S.J. 2019. The early Pliocene extinction of the mega-toothed shark Otodus megalodon: a view from the eastern North Pacific. PeerJ, 7:e6088. https://doi.org/10.7717/peerj.6088

Cappetta, H. 2012. Handbook of Paleoichthyology. Chondrichthyes (Mesozoic and Cenozoic Elasmobranchii: Teeth), vol. 3B. Gustav Fisher, Stuttgart, Germany.

Castro, J.I. 1993. The shark nursery of Bulls Bay, South Carolina, with a review of the shark nurseries of the southeastern coast of the United States. Environmental Biology of Fishes, 38:37-48. https://doi.org/10.1007/BF00842902

Cicimurri, D.J. and Knight, J.L. 2009. Late Oligocene sharks and rays from the Chandler Bridge Formation, Dorchester County, South Carolina, USA. Acta Palaeontologica Polonica, 54:627647. https://doi.org/10.4202/app.2008.0077 
Collareta, A., Lambert, O., Landini, W., Di Celma, C., Malinverno, E., Varas-Malca, R., Urbina, M., and Bianucci, G. 2017. Did the giant extinct shark Carcharocles megalodon target small prey? Bite marks on marine mammal remains from the late Miocene of Peru. Palaeogeography, Palaeoclimatology, Palaeoecology, 469:84-91. https://doi.org/10.1016/j.palaeo.2017.01.001

Cooper, J.A., Pimiento, C., Ferrón, H.G., and Benton, M.J. 2020. Body dimensions of the extinct giant shark Otodus megalodon: a 2D reconstruction. Scientific Reports, 10:14596. https://doi.org/10.1038/s41598-020-71387-y

Ehret, D.J. and Ebersole, J. 2014. Occurrence of the megatoothed sharks (Lamniformes: Otodontidae) in Alabama, USA. PeerJ, 2:e625. https://doi.org/10.7717/peerj.625

Erickson, B.R. 1990. Paleoecology of crocodile and whale-bearing strata of Oligocene age in North America. Historical Biology, 4:1-14. https://doi.org/10.1080/08912969009386530

Fallon, B.R. and Boessenecker, R.W. 2020. Multispecies leatherback turtle assemblage from the Oligocene Chandler Bridge and Ashley formations of South Carolina, USA. Acta Palaeontologia Polonica, 65:763-776. https://doi.org/10.4202/app.00740.2020

Föllmi, K.B. 1996. The phosphorus cycle, phosphogenesis and marine phosphate-rich deposits. Earth-Science Reviews, 40:55-124. https://doi.org/10.1016/0012-8252(95)00049-6

Frazzetta, T.H. 1988. The mechanics of cutting and the form of shark teeth (Chondrichthyes, Elasmobranchii). Zoomorphology, 108:93-107. https://doi.org/10.1007/BF00539785

Godfrey, S.J., Ellwood, M., Groff, S., and Verdin, M.S. 2018. Carcharocles-bitten odontocete caudal vertebrae from the coastal Eastern United States. Acta Palaeontologica Polonica, 63:463-468. https://doi.org/10.4202/app.00495.2018

Gottfried, M.D., Compagno, L.J.V., and Bowman, S.C. 1996. Size and skeletal anatomy of the giant "megatooth" shark Carcharodon megalodon, p. 55-66. In Klimley, A.P. and Ainley, D.G. (eds.), Great White Sharks: the Biology of Carcharodon carcharias. Academic Press, San Diego.

Gottfried, M.D. and Fordyce, R.E. 2001. An associated specimen of Carcharodon angustidens (Chondrichthyes, Lamnidae) from the Late Oligocene of New Zealand, with comments on Carcharodon interrelationships. Journal of Vertebrate Paleontology, 21:730-739. https://doi.org/10.1671/0272-4634(2001)021[0730:AASOCA]2.0.CO;2

Heithaus, M.R. 2007. Nursery areas as essential shark habitats: a theoretical perspective. American Fisheries Society Symposium, 50:3-13.

Herraiz, J.L., Ribé, J., Botella, H., Martínez-Pérez, C., and Ferrón, H.G. 2020. Use of nursery areas by the extinct megatooth shark Otodus megalodon (Chondrichthyes: Lamniformes). Biology Letters, 16:20200746. https://doi.org/10.1098/rsbl.2020.0746

Heupel, M.R., Carlson, J.K., and Simpfendorfer, C.A. 2007. Shark nursery areas: concepts, definition, characterization and assumptions. Marine Ecology Progress Series, 337:287-297. https://doi.org/10.3354/meps337287

Irschick, D.J. and Hammerschlag, N. 2015. Morphological scaling of body form in four shark species differing in ecology and life history. Biological Journal of the Linnean Society, 114:126-135. https://doi.org/10.1111/bij.12404

Irschick, D.J., Fu, A., Lauder, G., Wilga, G., and Hammerschlag, N. 2017. A comparative morphological analysis of body and fin shape for eight shark species. Biological Journal of the Linnean Society, 122:589-603. https://doi.org/10.1093/biolinnean/blx088

Kallal, R.J., Godfrey, S.J., and Ortner, D.J. 2010. Bone reactions on a Pliocene cetacean rib indicate short-term survival of predation event. International Journal of Osteoarchaeology, 22:253-260. https://doi.org/10.1002/oa.1199

Katuna, M.P., Geisler, J.H., and Colquhoun, D.J. 1997. Stratigraphic correlation of Oligocene marginal marine and fluvial deposits across the middle and lower coastal plain, South Carolina. Sedimentary Geology, 108:181-194. https://doi.org/10.1016/S0037-0738(96)00053-X

Kent, B.W. 2018. The cartilaginous fishes (chimaeras, sharks, and rays) of Calvert Cliffs, Maryland, USA. Smithsonian Contributions to Paleobiology, 100:45-157.

King, C., lakovleva, A., Steurbaut, E., Heilman-Clausen, C., and Ward, D.J. 2013. The Aktulagay section, west Kazakhstan: a key site for northern mid-latitude early Eocene stratigraphy. Stratigraphy, 10:171-209. 
Landini, W., Collareta, A., Pesci, F., Di Celma, C., Urbina, M., and Bianucci, G. 2017. A secondary nursery area for the copper shark Carcharhinus brachyurus from the late Miocene of Peru. Journal of South American Earth Sciences, 78:164-174. https://doi.org/10.1016/j.jsames.2017.07.003

McCuen, W.N., Ishimori, A.S., and Boessenecker, R.W. 2020. A new specimen of Xiphiorhynchus cf. $X$. aegyptiacus (Istiophoriformes, Xiphiodei, Xiphiidae) and billfish diversity in the Oligocene of South Carolina. Vertebrate Anatomy Morphology Paleontology, 8:98-104. https://doi.org/10.18435/vamp29367

Perez, V.J., Godfrey, S.J., Kent, B.W., Weems, R.E., and Nance, J.R. 2019. The transition between Carcharocles chubutensis and Carcharocles megalodon (Otodontidae, Chondrichthyes): lateral cusplet loss through time. Journal of Vertebrate Paleontology, 38:e1546732. https://doi.org/10.1080/02724634.2018.1546732

Perez, V.J., Leder, R.M., and Badaut, T. 2021. Body length estimation of Neogene macrophagous lamniform sharks (Carcharodon and Otodus) derived from associated fossil dentitions. Palaeontologia Electronica, 24:a09. https://doi.org/10.26879/1140.

Pimiento, C., Ehret, D.J., MacFadden, B.J., and Hubbell, G. 2010. Ancient nursery area for the extinct giant shark Megalodon from the Miocene of Panama. PLoS ONE, 5:e10552. https://doi.org/10.1371/journal.pone.0010552

Purdy, R.W. 1996. Paleoecology of fossil white sharks, p. 67-78. In Klimley, A.P. and Ainley, D.G. (eds.), Great White Sharks: The Biology of Carcharodon carcharias. Academic Press, San Diego.

Purdy, R.W., Schneider, V.P., Applegate, S.P., McLellan, J.H., Meyer, R.L., and Slaughter, B.H. 2001. The Neogene sharks, rays, and bony fishes from the Lee Creek Mine, Aurora, North Carolina. Smithsonian Contributions to Paleobiology, 90:71-202.

Sanders, A.E. 1980. Excavation of Oligocene marine fossil beds near Charleston, South Carolina. National Geographic Research Reports, 12:601-621.

Sanders, A.E., Weems, R.E., and Lemon, E.M., Jr. 1982. Chandler Bridge Formation - a new Oligocene stratigraphic unit in the lower coastal plain of South Carolina. US Geological Survey Bulletin, 1529-H:H105-H124.

Shimada, K. 2002. The relationship between the tooth size and total body length in the white shark, Carcharodon carcharias (Lamniformes: Lamnidae). Journal of Fossil Research, 35:28-33.

Shimada, K., Chandler, R.E., Lam, O.L.T., Tanaka, T., and Ward, D.J. 2017. A new elusive otodontid shark (Lamniformes: Otodontidae) from the lower Miocene, and comments on the taxonomy of otodontid genera, including the 'megatoothed' clade. Historical Biology, 29:704714. https://doi.org/10.1080/08912963.2016.1236795

Shimada, K., Becker, M.A., and Griffiths, M.L. 2020. Body, jaw, and dentition lengths of macrophagous lamniform sharks, and body size evolution in Lamniformes with special reference to 'off-the-scale' gigantism of the megatooth shark, Otodus megalodon. Historical Biology, online early. https://doi.org/10.1080/08912963.2020.1812598

Villafaña, J.A., Hernandez, S., Alvarado, A., Shimada, K., Pimiento, C., Rivadeneira, M.M., and Kriwet, J. 2020. First evidence of a palaeo-nursery area of the great white shark. Scientific Reports, 10:8502. https://doi.org/10.1038/s41598-020-65101-1

Weems, R.E., Bybell, L.M., Edwards, L.E., Lewis, W.C., Self-Trail, J.M., Albright, L.B., III,, Cicimurri, D.J., Harris, W.B., Osborne, J.E., and Sanders, A.E. 2016. Stratigraphic revision of the Cooper Group and Chandler Bridge and Edisto Formations in the coastal plain of South Carolina. South Carolina Geology, 49:1-24.

Zhelezko, V. and Kozlov, V. 1999. Elasmobranchii and Palaeogene biostratigraphy of Transurals and Central Asia. Materials on Stratigraphy and Palaeontology of the Urals, 3:1-324. 